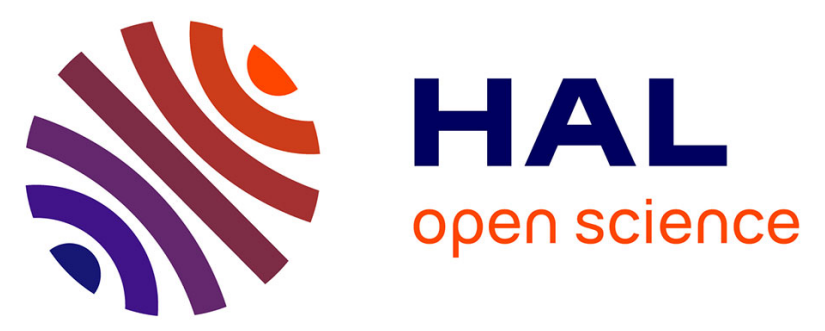

\title{
CONSTANTES OPTIQUES DE SEPT CRISTAUX IONIQUES A BASSE TEMPÉRATURE DANS L'INFRAROUGE LOINTAIN, PROCESSUS DE DIFFÉRENCE, ROLE DES IMPURETÉS
}

\author{
A. Hadni, P. Henry, J.-P. Lambert, G. Morlot, P. Strimer, D. Chanal
}

\section{To cite this version:}

A. Hadni, P. Henry, J.-P. Lambert, G. Morlot, P. Strimer, et al.. CONSTANTES OPTIQUES DE SEPT CRISTAUX IONIQUES A BASSE TEMPÉRATURE DANS L'INFRAROUGE LOINTAIN, PROCESSUS DE DIFFÉRENCE, ROLE DES IMPURETÉS. Journal de Physique Colloques, 1967, 28 (C1), pp.C1-118-C1-128. 10.1051/jphyscol:1967115 . jpa-00213169

HAL Id: jpa-00213169 https://hal.science/jpa-00213169

Submitted on 1 Jan 1967

HAL is a multi-disciplinary open access archive for the deposit and dissemination of scientific research documents, whether they are published or not. The documents may come from teaching and research institutions in France or abroad, or from public or private research centers.
L'archive ouverte pluridisciplinaire HAL, est destinée au dépôt et à la diffusion de documents scientifiques de niveau recherche, publiés ou non, émanant des établissements d'enseignement et de recherche français ou étrangers, des laboratoires publics ou privés. 


\title{
CONSTANTES OPTIQUES DE SEPT CRISTAUX IONIQUES A BASSE TEMPÉRATURE DANS L'INFRAROUGE LOINTAIN, PROCESSUS DE DIFFÉRENCE, ROLE DES IMPURETÉS
}

\author{
par A. Hadni, P. Henry, J.-P. Lambert, G. Morlot, P. Strimer et D. Chanal \\ Institut de Physique, Faculté des Sciences, Nancy.
}

\begin{abstract}
Résumé. - Détermination des constantes optiques de $\mathrm{AgCl}, \mathrm{AgBr}, \mathrm{AgI}, \mathrm{ZnSe}, \mathrm{ZnTe}, \mathrm{CdSe}$ entre $300^{\circ} \mathrm{K}$ et $30^{\circ} \mathrm{K}$, à partir des spectres de réflexion. Dans certains cas (ZnSe), l'amortissement diminue de façon spectaculaire lorsqu'on refroidit. Etude de la supertransmission de $\mathrm{KBr}$ dans l'Infrarouge lointain, à basse température : le coefficient d'absorption entre 161 et 349 microns varie comme la Température absolue $T$, ce qui confirmerait une interprétation par des processus de différence à deux phonons. Toutefois, la précision des mesures est faible et ne permet pas d'éliminer la possibilité de transitions faisant intervenir plus de deux phonons. A basse température, des bandes fines se dégagent parfois du fond continu et sont attribuées à des impuretés.
\end{abstract}

\begin{abstract}
The optical constants of $\mathrm{AgCl}, \mathrm{AgBr}, \mathrm{AgI}, \mathrm{ZnSe}, \mathrm{ZnTe}, \mathrm{CdSe}$ at temperatures ranging from $300^{\circ} \mathrm{K}$ down to $30^{\circ} \mathrm{K}$ are determined from reflexion spectra. For $\mathrm{ZnSe}$ the damping diminishes drastically with cooling. A quantitative study is made of the high transmission of $\mathrm{KBr}$ at low temperature : the absorption coefficient increases as $T$ between 161 and 349 microns. This confirms a two phonon difference process interpretation. However, the accuracy is low and it is not possible to reject some contribution from three phonon transitions. At low temperature sharp bands are found in commercial samples containing impurities.
\end{abstract}

I. Introduction. - L'absorption des cristaux dans l'Infrarouge lointain, au-delà de la dernière résonance ionique du cristal, a fait l'objet de plusieurs études ces dernières années et des explications apparaissent. Le fait le plus important est sans doute la transparence extraordinaire des cristaux à la température de l'hélium liquide, alors qu'à $300^{\circ} \mathrm{K}$, l'absorption est presque toujours considérable [1]. L'intervention de transitions de différence à plusieurs phonons explique cet effet [2], mais dans l'hypothèse d'un oscillateur linéaire, l'amortissement des vibrations joue aussi un rôle important, et des mesures quantitatives sont nécessaires pour distinguer la part des différents processus. Par ailleurs, lorsque par refroidissement, on a supprimé cette absorption, des bandes faibles, dues à des impuretés, apparaissent alors et sont encore bien peu connues [3]. Ces problèmes difficiles et nouveaux seront considérés aux paragraphes $\mathrm{V}$ à IX et nous commencerons par des exercices plus classiques : la détermination des constantes optiques des halogénures d'argent et de trois composés II-V au voisinage de la résonance à partir des spectres de réflexion que nous avons obtenus pour la première fois dans un domaine étendu de températures et sous faible incidence. Ils permettent d'évaluer la diminution de l'amortissement lorsque la température diminue.

II. Expérimentation. - Les coefficients d'absorption et de réflexion dans l'infrarouge lointain sont obtenus par spectrométrie à réseau. La limite de résolution peut être inférieure à un nombre d'ondes par $\mathrm{cm}^{-1}$. Des cryostats permettent d'obtenir les spectres d'absorption et de réflexion à des températures descendant au-dessous de $4{ }^{\circ} \mathrm{K}$. La température est mesurée par un bolomètre au carbone logé dans une cavité creusée dans l'échantillon ou dans son support de quartz (Fig. 1). Différents points fixes sont réalisés en plaçant l'échantillon sur une carotte de cuivre en contact avec différents fluides cryogéniques usuels : azote, hydrogène, hélium liquides, avec ou sans écrans thermiques. Dans ce premier cas, les températures mesurées sont, par exemple, $90 \circ \mathrm{K}, 32 \circ \mathrm{K}, 19 \circ \mathrm{K}$ respectivement. L'échantillon peut également être immergé directement dans les différents fluides, le rayonnement arrive alors par une fenetre de quartz en contact avec le réservoir cryogénique par l'intermédiaire d'un joint d'indium. Avant chaque spectre, l'étanchéité doit être contrôlée au spectromètre de masse. Dans le cas 


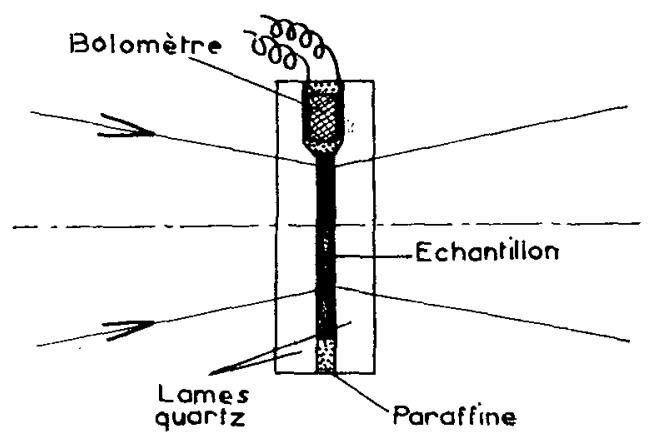

Fig. 1. - Bolomètre pour la mesure de la température du cristal étudié.

de la réflexion, l'incidence est de $7,5^{\circ}$, et les constantes optiques sont déterminées par une analyse de KramersKronig d'abord, une analyse de Lorentz à une ou deux résonances ensuite $\left({ }^{1}\right)$.

III. Spectres de réflexion des halogénures d'argent à basse température. - Les figures 2 et 3 correspondent

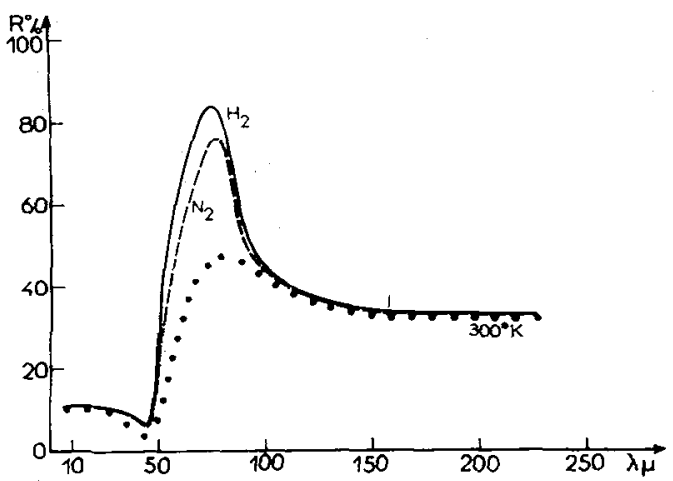

Fig. 2. - Pouvoir réflecteur du chlorure d'argent à trois températures.

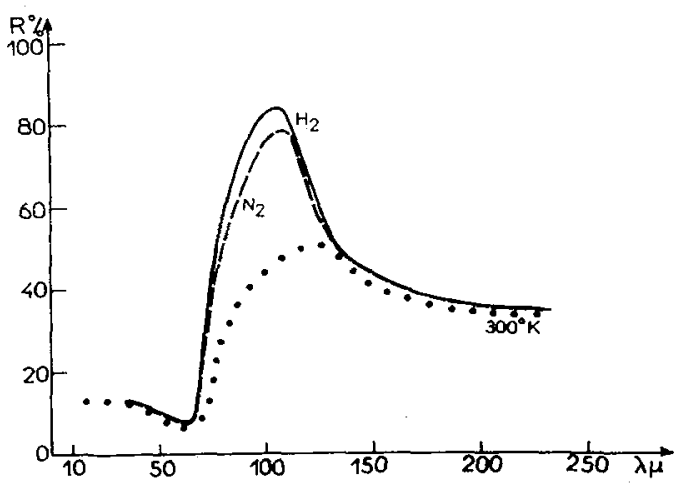

FIG. 3. - Pouvoir réflecteur du bromure d'argent à trois températures.

(1) Les résuitats de l'analyse de Lorentz ne sont pas donnés dans ce mémoire. respectivement à des monocristaux de chlorure et de bromure d'argent, qui cristallisent dans le système cubique du type $\mathrm{NaCl}$ : le spectre de réflexion se précise de façon similaire lorsqu'on refroidit les deux cristaux.

Les figures 4, 5, 6 donnent les constantes optiques
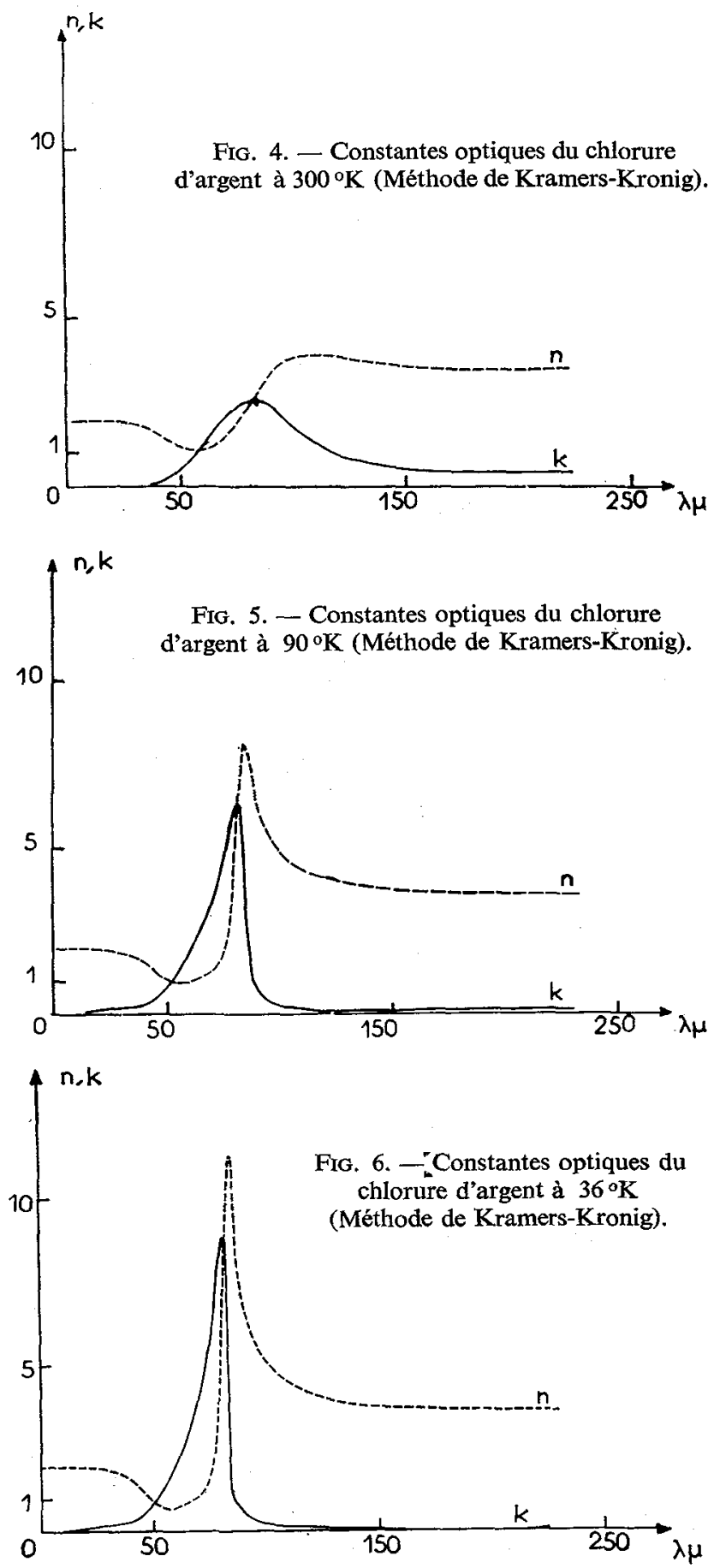


\section{C $1-120$}

A." HADNI,IP. HENRI, J.-P. LAMBERT,

du chlorure qd'argent à $300 \% \mathrm{~K}$ et aux températures de l'azote et de l'hydrogène liquides respectivement. Les figures $7,8,9{ }_{\AA}$ correspondent, de même, au bromure d'argent.
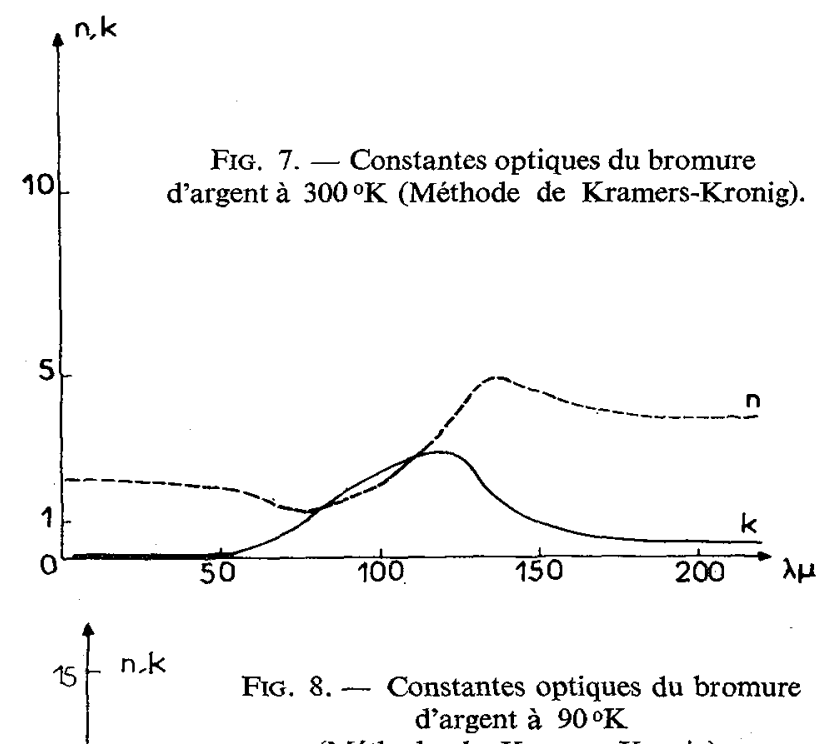
(Méthode de Kramers-Kronig).
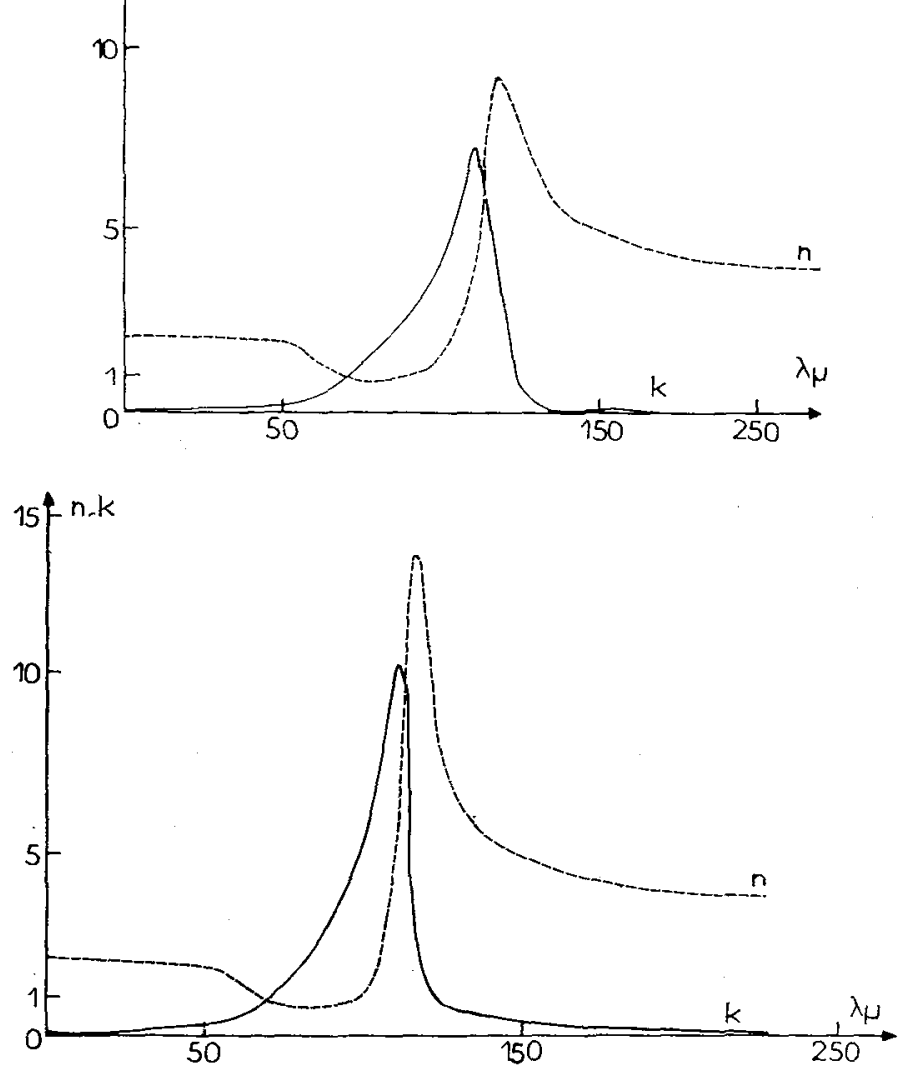

FIG. 9. - Constantes optiques du bromure d'argent à $36^{\circ} \mathrm{K}$ (Méthode de Kramers-Kronig).
Dans tous les cas, la bande d'absorption voit sa largeur diminuer, et son maximum croître dans un rapport de l'ordre de 3 lorsqu'on refroidit.

Les figures 10,11, 12 donnent les évolutions de $\delta=\Delta \lambda / \lambda_{T}$ (facteur d'amortissement), de $v_{T}$ (fréquence de résonance) et de $n_{0}$ (indice de réfraction limite

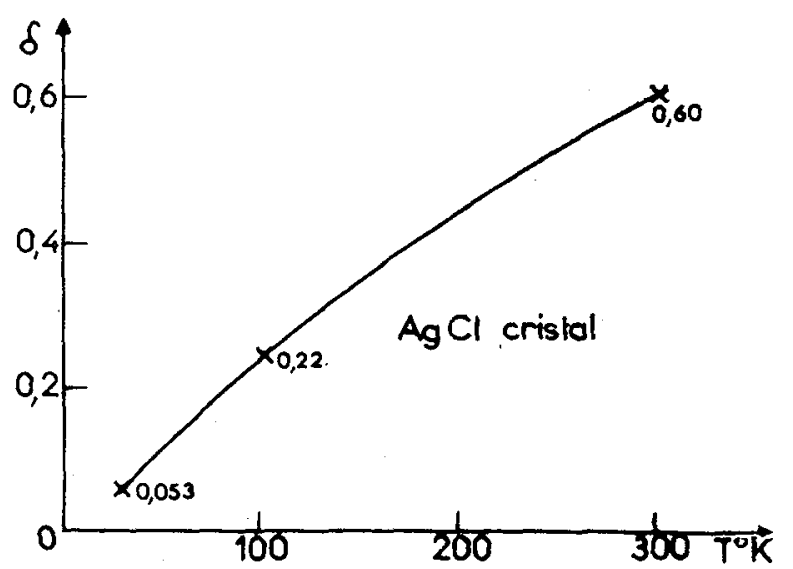

FIG. 10. - Constante d'amortissement du chlorure d'argent en fonction de la température.
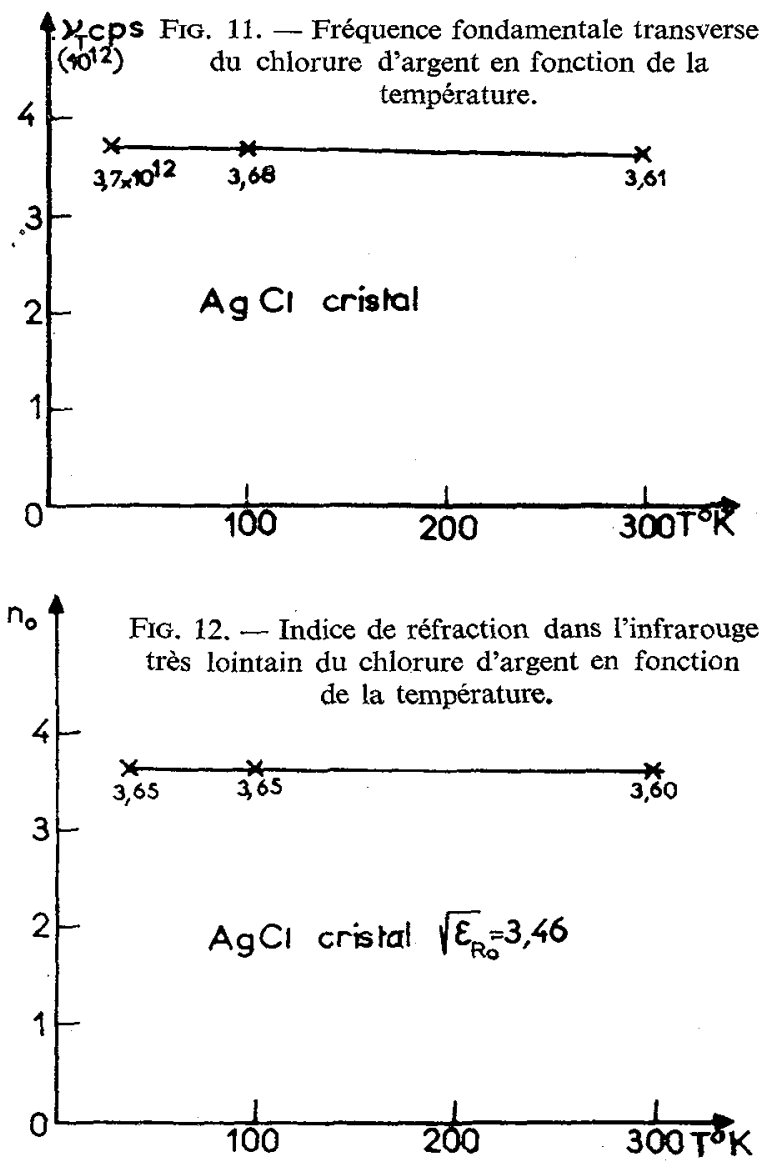


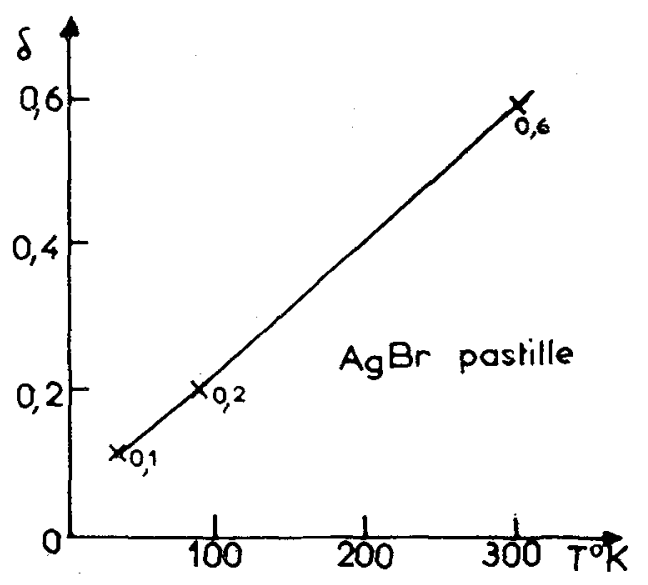

Fig. 13. - Constante d'amortissement du bromure d'argent en fonction de la température.

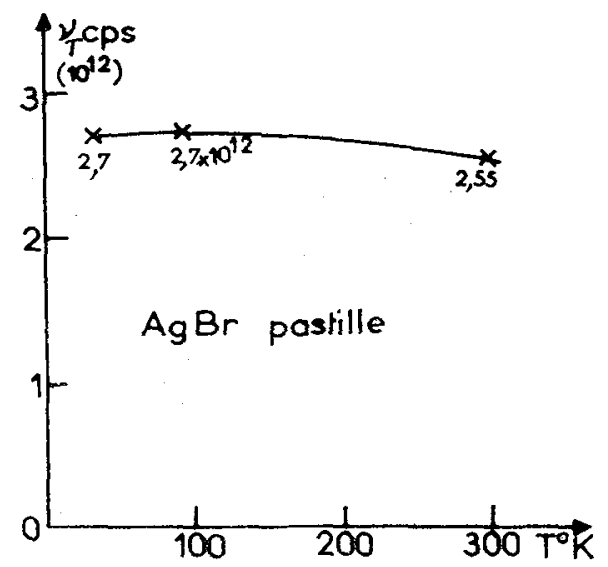

Fig. 14. - Fréquence fondamentale transverse du bromure d'argent en fonction de la température.

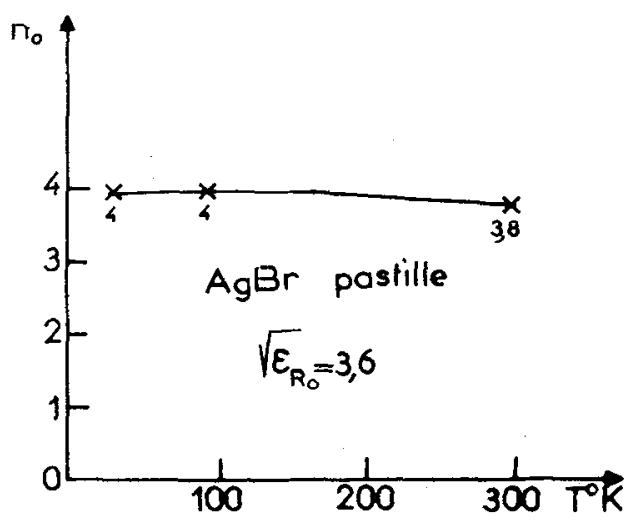

FIG. 15. - Inđice de réfraction dans l'infrarouge très lointain du bromure d'argent en fonction de la température. pour les basses fréquences) en fonction de la température pour le chlorure d'argent. On rappelle que $\sqrt{\varepsilon_{\mathrm{R} 0}}=3,46$ à $300 \mathrm{~K}$. Les figures $13,14,15$ correspondent de même au bromure d'argent. Ici,

$$
\sqrt{\varepsilon_{\mathrm{R} 0}}=3,6 \text { à } 300 \mathrm{oK} \text {. }
$$

$\mathrm{AgCl}$ et $\mathrm{AgBr}$ avaient déjà été étudiés par Martin [4], en transmission. Pour $\mathrm{AgCl}$ nous trouvons un amortissement 3 fois plus grand à basse température. Par contre, l'accord est meilleur avec une analyse de Lorentz, comme nous le montrerons dans un prochain mémoire. En ce qui concerne l'iodure d'argent (Fig. 16), on sait qu'il se trouve dans la nature sous la

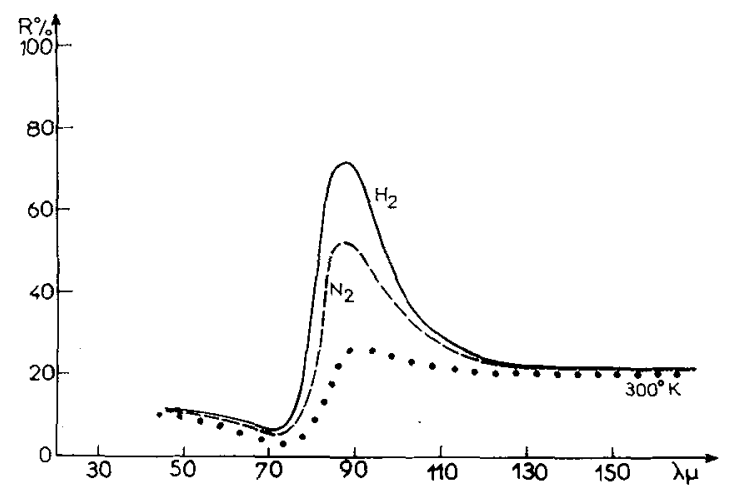

FIG. 16. - Pouvoir réflecteur de l'iodure d'argent à trois températures.

forme d'iodargyrite, hexagonale et pseudo-cubique comme la wurtzite. C'est sous cette forme qu'on l'obtient par fusion. Les figures 17, 18, 19 donnent les

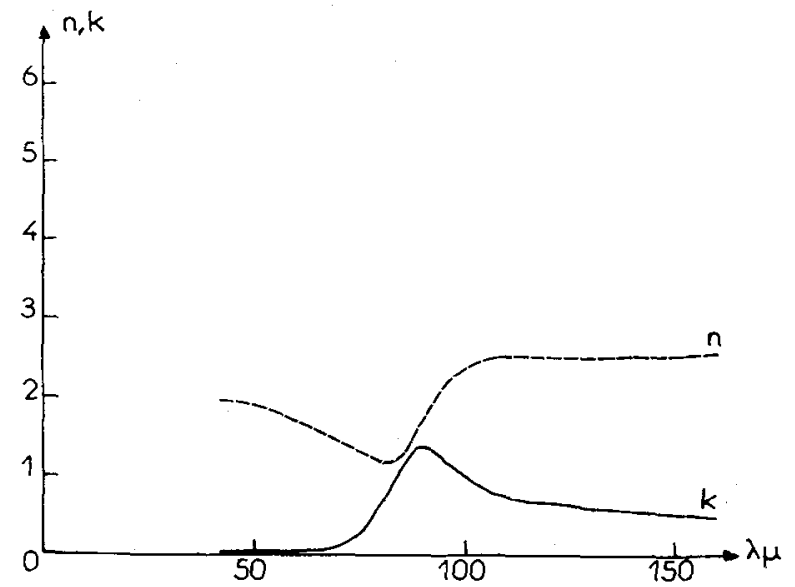

Fig. 17. - Constantes optiques de l'iodure d'argent à $300^{\circ} \mathrm{K}$ (Méthode de Kramers-Kronig). 


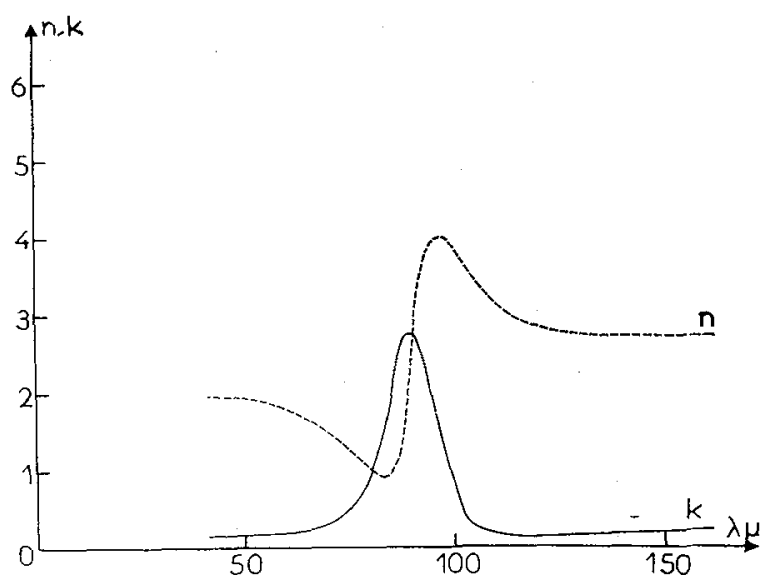

Frg. 18. - Constantes optiques de l'iodure d'argent à $90^{\circ} \mathrm{K}$ (Méthode de Kramers-Kronig).

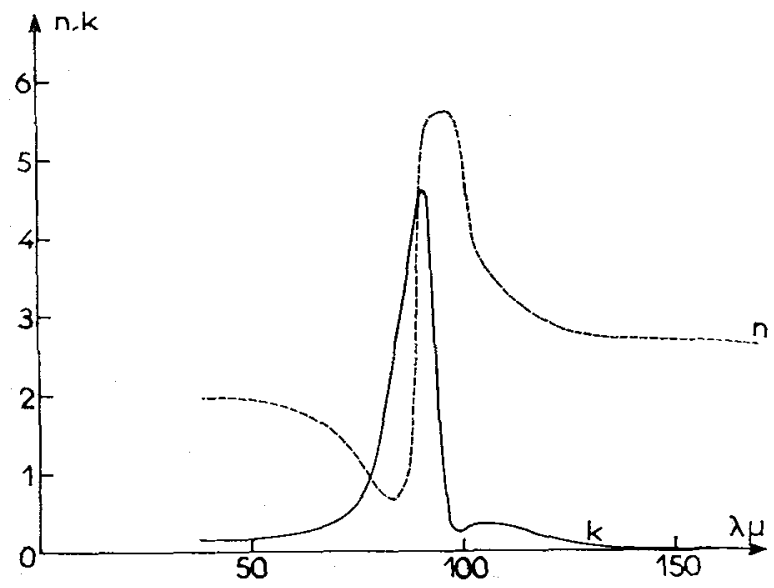

Frg. 19. - Constantes optiques de l'iodure d'argent à $36^{\circ} \mathrm{K}$ (Méthode de Kramers-Kronig).

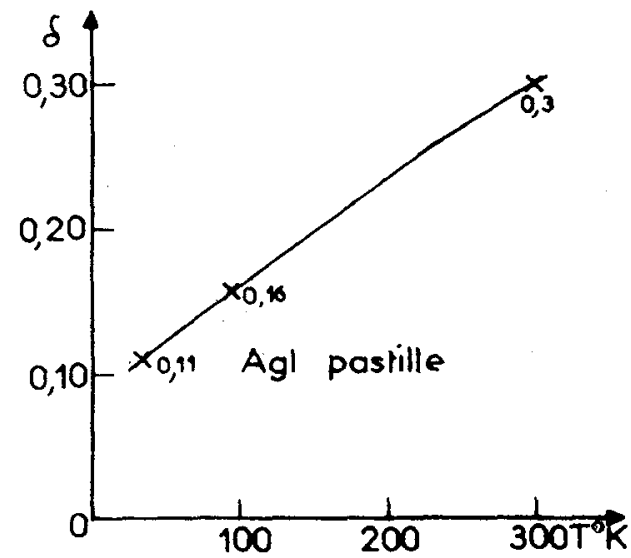

FIg. 20. - Constante d'amortissement de l'iodure d'argent en fonction de la température.

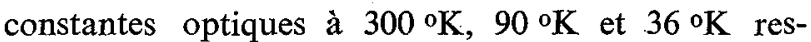
pectivement. Les figures 20,21 et 22 montrent une certaine évolution du coefficient d'amortissement avec la température, par contre $v_{\mathrm{T}}$ et $n_{0}$ varient peu.
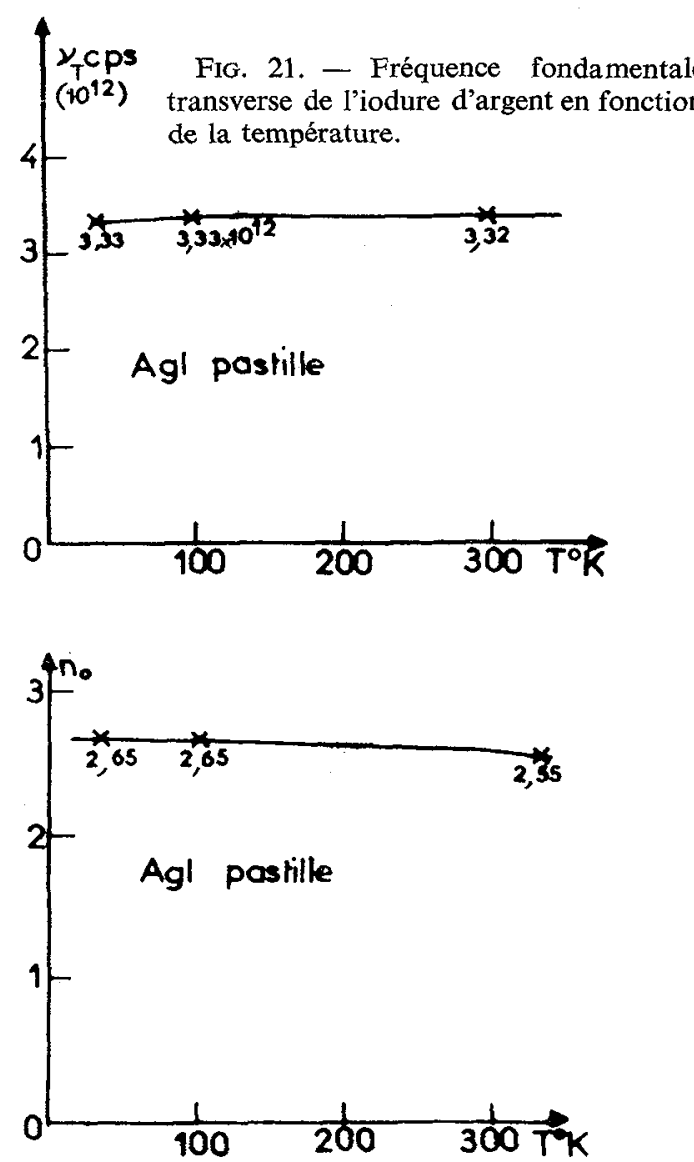

Fig. 22, - Indice de réfraction dans l'infrarouge très lointain de l'iodure d'argent en fonction de la température.

IV. Spectres de réflexion des composés II-V à basse température. - Les figures 23,24, 25 correspondent

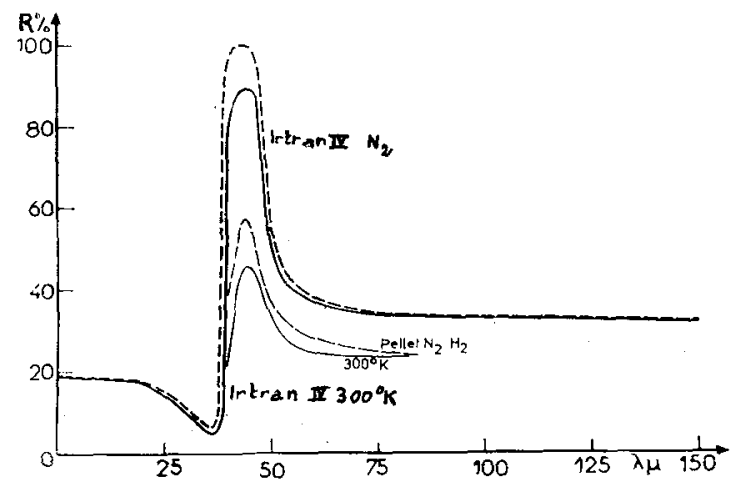

Fig. 23. - Pouvoir réflecteur de ZnSe à trois températures 


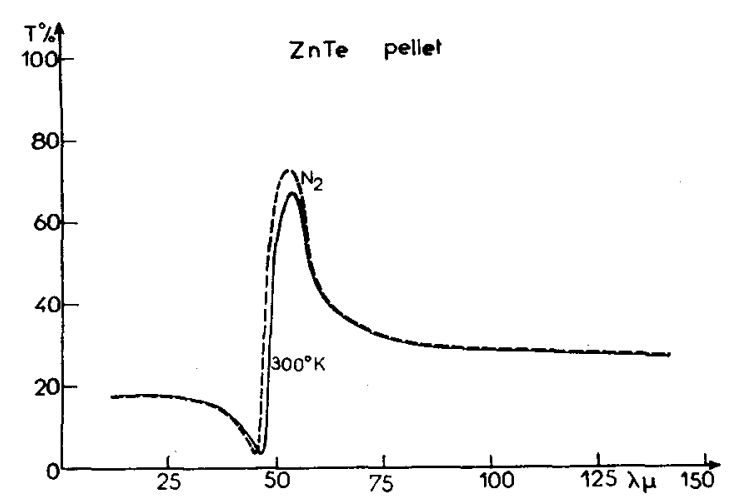

FIG. 24. - Pouvoir réflecteur de ZnTe à deux températures.

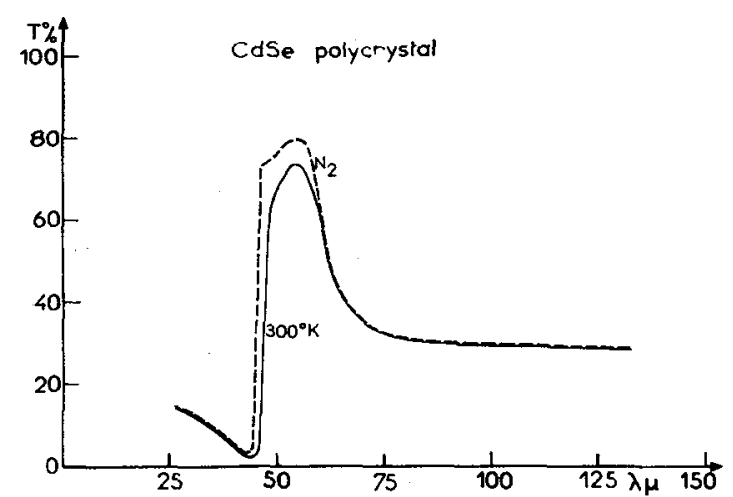

FIG. 25. - Pouvoir réflecteur de CdSe à deux températures.

respectivement à $\mathrm{ZnSe}, \mathrm{ZnTe}$ et CdSe. L'influence du refroidissement est moins sensible que précédemment, car à la température ordinaire, particulièrement pour $\mathrm{ZnSe}$, le coefficient de réflexion atteint $90 \%$ et dès la température de l'azote liquide, il rejoint la valeur limite de $100 \%$ correspondant à l'harmonicité parfaite. Les figures 26 et 27 , donnent les constantes optiques de $\mathrm{ZnSe}$ à $300 \circ \mathrm{K}$ et $90 \circ \mathrm{K}$ respectivement, et les figures 28 et 29

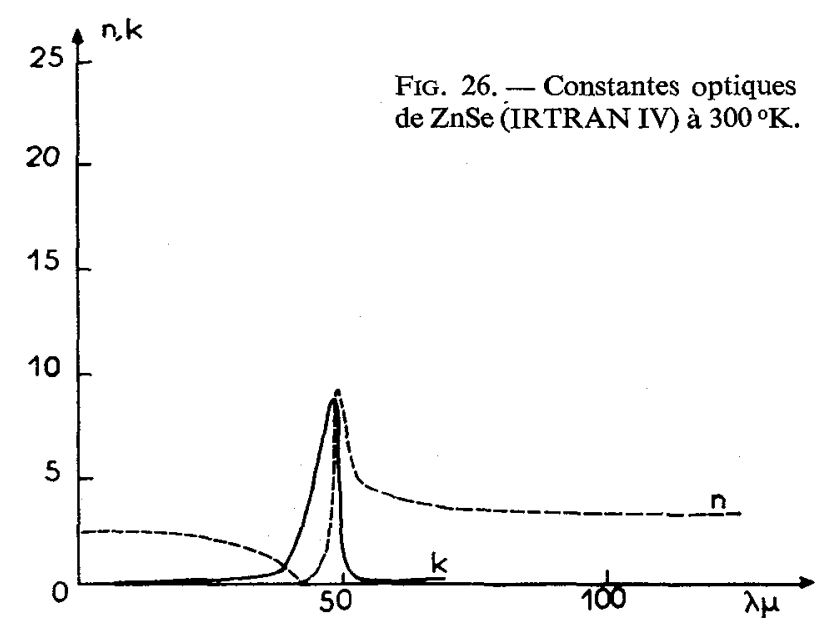

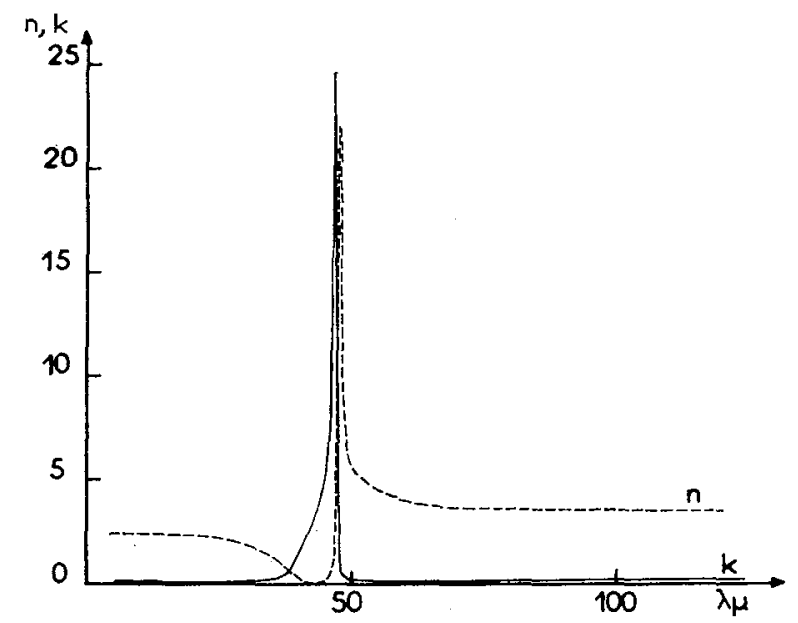

Fig. 27. - Constantes optiques de ZnSe (IRTRAN IV) à $90^{\circ} \mathrm{K}$.

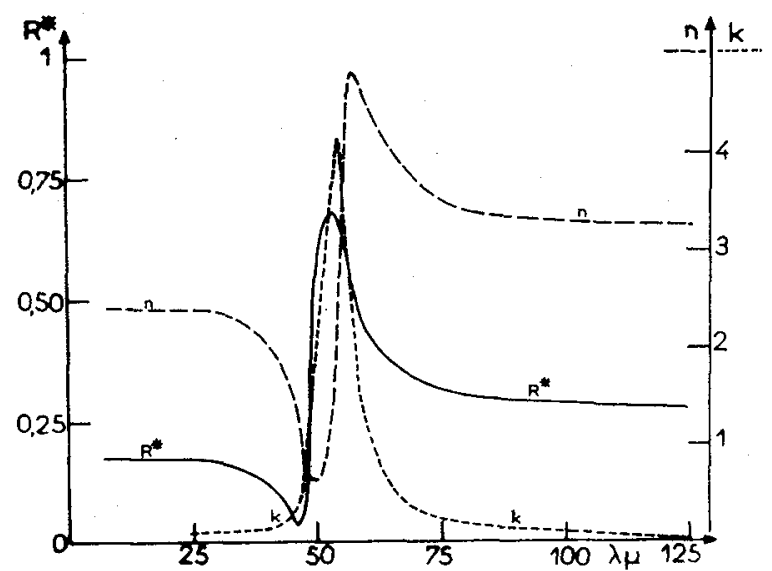

FIG, 28. - Constantes optiques de ZnTe (pastille) à $300^{\circ} \mathrm{K}$, $n_{\mathrm{v}}=2,43 ; n_{0}=3,17 ; \lambda_{\mathrm{T}}=54,5 \mu ; \delta=0,21$.

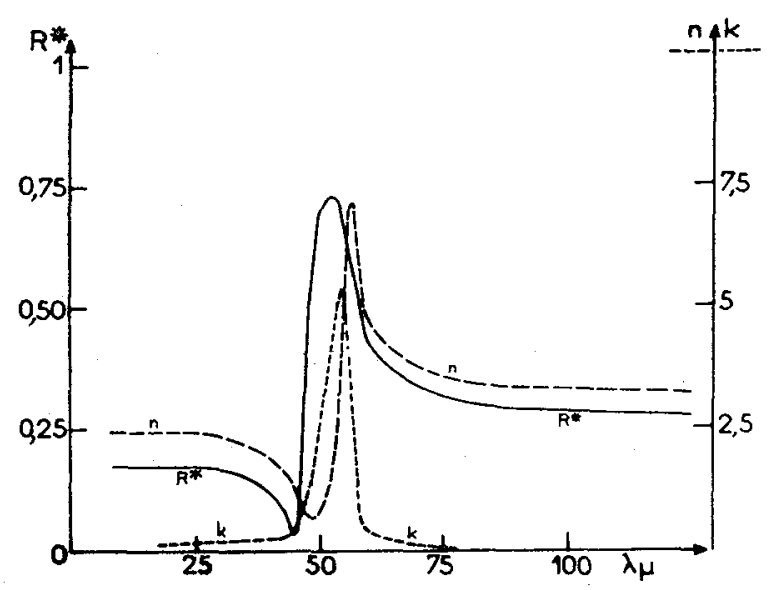

Fig. 29. - Constantes optiques de ZnTe (pastille) à $90 \% \mathrm{~K}$, $n_{\mathrm{v}}=2,43 ; n_{0}=3,16 ; \lambda_{\mathrm{T}}=54,5 \mu ; \delta=0,11$. 
correspondent de même à $\mathrm{ZnTe}$. Les figures 30 et 31 se rapportent à $\mathrm{CdSe}$. La figure 32 donne essentiellement le coefficient d'amortissement $\delta$ de l'Irtran 4 en fonction de la température, l'amortissement est extraordinairement faible à basse température. Ces composés avaient déjà été étudiés par Mitsuishi [8,9], nous trouvons des longueurs d'onde un peu plus petites, des amortissements plus grands à température ordinaire, et du même ordre de grandeur à basse température.

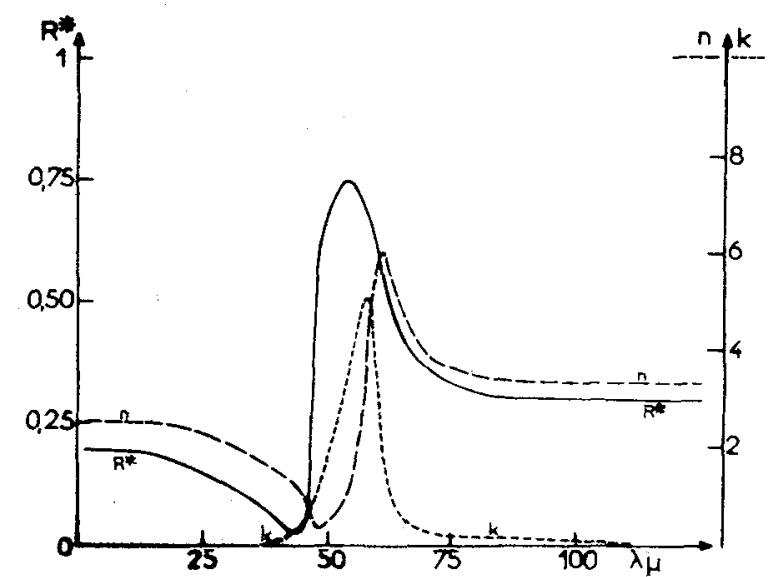

FIG. 30. - Constantes optiques de CdSe (pastille) à $300^{\circ} \mathrm{K}$, $n_{\vee}=2,56 ; n_{0}=3,31 ; \lambda_{\mathrm{T}}=58 \mu ; \delta=0,16$.

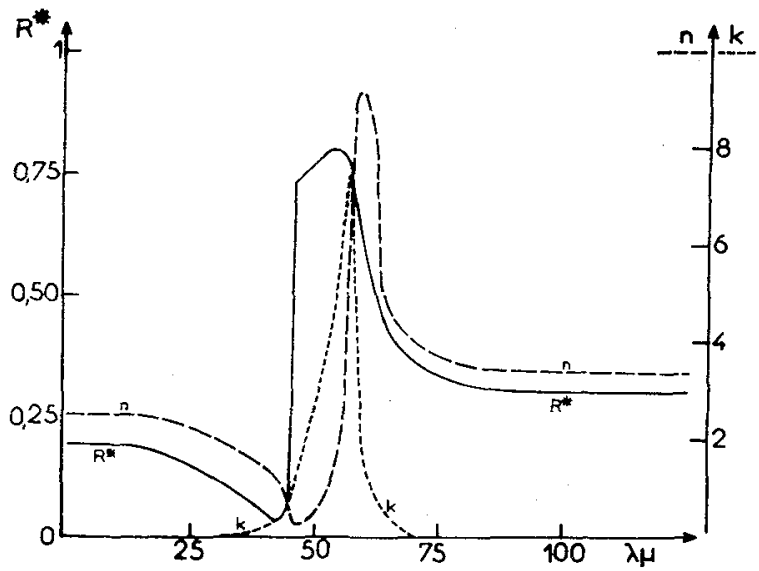

FIG. 31. - Constantes optiques de CdSe (pastille) à $90^{\circ} \mathrm{K}$, $n_{\mathrm{v}}=2,56 ; n_{0}=3,3 ; \lambda_{\mathrm{T}}=57,5 \mu ; \delta=0,12$.

V. Transmission du bromure de patassium pour 5 longueurs d'onde remarquables. - La figure 33 représente 4 isochromes du Bromure de Potassium, obtenues à partir d'une série de spectres de transmission (Fig. 34). Les courbes sont sensiblement des droites, de pente décroissante lorsque $\lambda$ augmente.

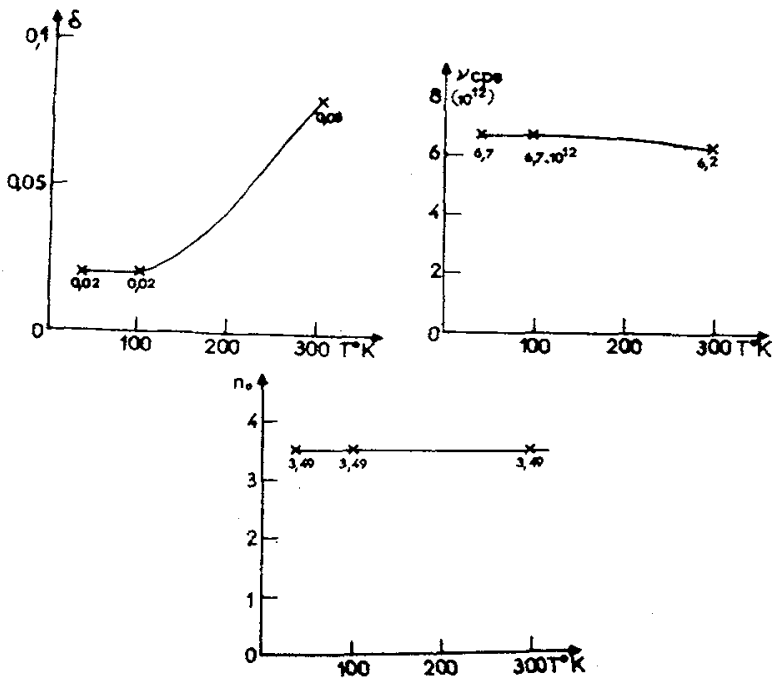

FIG. 32. - Variations du coefficient d'amortissement $\delta$ de la fréquence fondamentale transversale $v_{T}$, et de l'indice de réfraction $n_{0}$ pour les très grandes longueurs d'onde, en fonction de la température, pour l'Irtran IV (ZnSe).

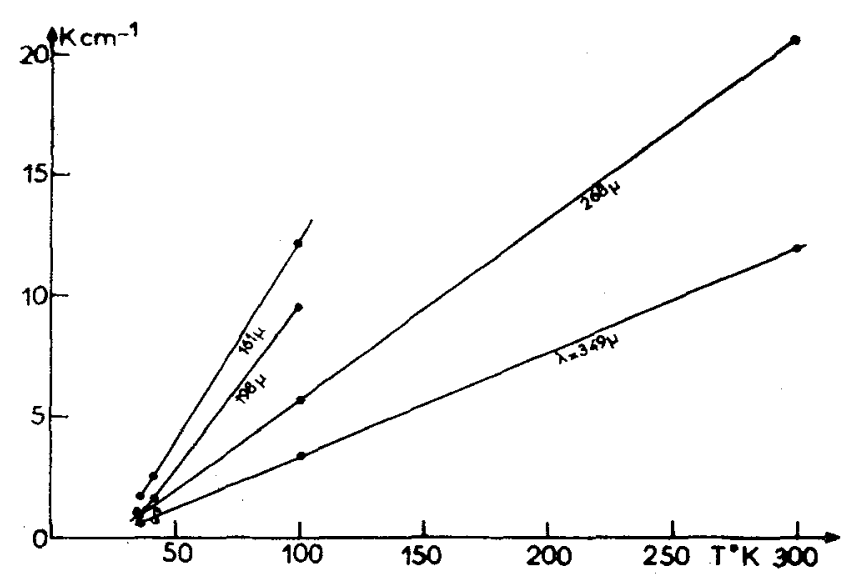

FIG. 33. - Coefficient d'absorption $K$ du bromure de potassium en fonction de la température absolue $T$ pour quatre longueurs d'onde caractéristiques.

VI. Rôle de l'amortissement de la vibration fondamentale d'un cristal dans l'absorption de l'infrarouge lointain. - Dans le cadre de la théorie de Lorentz (oscillateurs linéaires amortis de fréquence $\omega_{1}$ ), les parties réelle $\varepsilon^{\prime}$ et imaginaire $\varepsilon^{\prime \prime}$ de la constante diélectrique s'écrivent :

$$
\begin{aligned}
& \varepsilon^{\prime}=n^{2}-k^{2}=\varepsilon_{\mathrm{V}}+\left(\varepsilon_{\mathrm{R} 0}-\varepsilon_{\mathrm{V}}\right) \frac{1-\Omega^{2}}{\left(1-\Omega^{2}\right)^{2}+\delta^{2} \Omega^{2}} \\
& \varepsilon^{\prime \prime}=2 n k=\left(\varepsilon_{\mathrm{R} 0}-\varepsilon_{\mathrm{V}}\right) \frac{\Omega \delta}{\left(1-\Omega^{2}\right)^{2}+\delta^{2} \Omega^{2}} .
\end{aligned}
$$




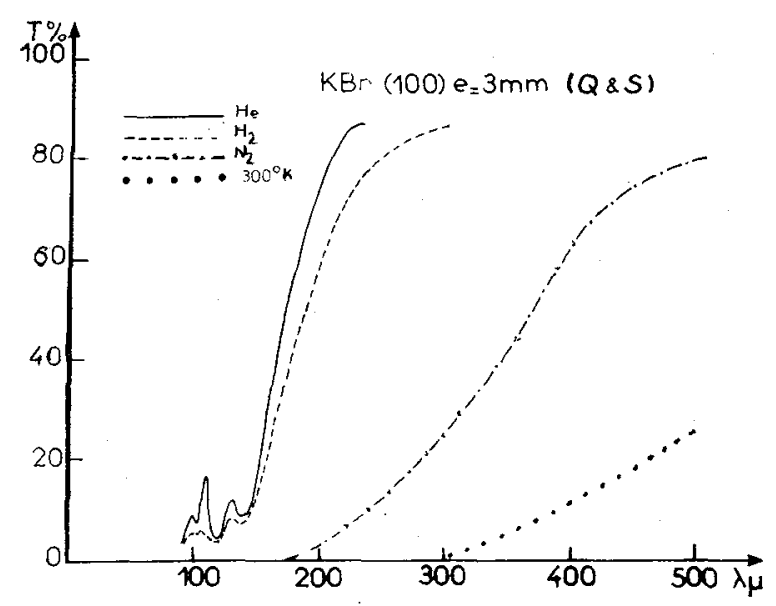

FIG. 34. - Transmission d'une lame de bromure de potassium à quatre températures différentes.

Dans la théorie la plus simple, $\delta$ est une constante d'amortissement indépendante de la fréquence, et $\Omega=\omega / \omega_{1}$. On en déduit l'indice d'absorption

$$
k \simeq \frac{\left(\varepsilon_{\mathrm{R} 0}-\varepsilon_{\mathrm{V}}\right) \Omega \delta}{2\left[\left(1-\Omega^{2}\right)^{2}+\Omega^{2} \delta^{2}\right]}
$$

pour $\Omega<0,7$ environ. On voit que, dans l'infrarouge lointain, $k$ est proportionnel à $\delta$ et diminue en même temps que $\Omega$. Pour $\mathrm{KBr}$ à $300 \mathrm{oK}$, Martin [4] propose $\delta=0,058$. D'où le tableau I donnant $k$ et le coefficient d'absorption $\mathrm{K}$ pour quatre longueurs d'onde particulières.

\section{TABleau I}

Contribution de l'amortissement au coefficient d'absorption de $\mathrm{KBr}$ à $300^{\circ} \mathrm{K}$.

\begin{tabular}{|c|c|c|c|c|}
\hline$\lambda$ & $349 \mu$ & $268 \mu$ & $161 \mu$ & $122 \mu$ \\
\hline$k$ & $\begin{array}{c}0,9 \times 10^{-2} \\
3,22 \mathrm{~cm}^{-1}\end{array}$ & $\begin{array}{c}1,29 \times 10^{-2} \\
6,05 \mathrm{~cm}^{-1}\end{array}$ & $\begin{array}{c}3,15 \times 10^{-2} \\
24,6 \mathrm{~cm}^{-1}\end{array}$ & $103 \mathrm{~cm}^{-1}$ \\
\hline
\end{tabular}

On voit sur la figure 33 que les coefficients d'absorption mesurés à température ordinaire sont très supérieurs à cette contribution, mais qu'elle n'est toutefois pas négligeable. Lorsqu'on refroidit le cristal, la contribution de l'amortissement à l'absorption diminue comme $\delta$. A 122 microns par exemple, l'absorption résiduelle mesurée est de l'ordre de $10 \mathrm{~cm}^{-1}$ à $32 \circ \mathrm{K}$, il faudrait donc supposer qu'à cette température $\delta$ est divisé au moins par 10. Martin ne donne aucune valeur pour $\delta$ à cette température, mais une telle variation n'est pas commune. En résumé, on peut dire que la théorie classique de Lorentz est insuffisante pour expliquer l'absorption considérable des cristaux dans l'infrarouge lointain à température ordinaire et, qu'à basse température au contraire, si elle explique qualitativement l'augmentation de la transparence, elle est toutefois incapable de prévoir l'importance du phénomène à partir de l'affinissement, assez limité en général, de la bande d'absorption, lorsqu'on diminue la température.

VII. Rôles des processus de différence à deux phonons . - VII.1 CALCUL DU COEFFICIENT D'ABSORPTION. On sait que les processus de différence interviennent dans l'hypothèse où les oscillateurs ne sont plus linéaires, ou lorsqu'on considère un terme du second ordre dans la variation du moment dipolaire électrique du vibrateur. Nous considérons d'abord le cas le plus simple où un phonon $v_{1}$ disparaît et donne naissance à un phonon plus énergétique $v_{2}$ grâce à l'apport d'énergie $h v$ du photon d'infrarouge lointain. D'une part $v_{2}-v_{1}=v$ pour conserver l'énergie, d'autre part il s'agit de transitions ( verticales ) pour conserver la quantité de mouvement (le vecteur d'onde du photon est négligeable vu la grande longueur d'onde des radiations infrarouges par rapport à celles de la plupart des ondes d'agitation thermique). On montre [5] que le coefficient d'absorption $K\left(\mathrm{~cm}^{-1}\right)$ s'écrit :

$K=B \frac{\omega}{n} \frac{\varepsilon_{\mathrm{R} 0}-n_{\mathrm{V}}^{2}}{\left(\omega^{2}-\omega_{\mathrm{T}}^{2}\right)^{2}} \sum_{\omega_{2}>\omega_{1}} \frac{n_{1}-n_{2}}{\omega_{1} \omega_{2}} \delta\left(\omega_{2}-\omega_{1}-\omega\right)$

avec

$$
n_{1}-n_{2}=\frac{1}{\mathrm{e}^{h v_{1} / k T}-1}-\frac{1}{\mathrm{e}^{h v_{2} / k T}-1} .
$$

Nous voyons que, pour une longueur d'onde donnée, $K$ est une fonction de $T$ qui peut être très simple dans le cas où l'absorption de $v$ ne fait intervenir principalement que deux types de phonons, de fréquences très voisines de $v_{1}$ et de $v_{2}$. C'est peut-être ce qui se passe pour les combinaisons de phonons caractéristiques correspondant aux extrémités de la zone de Brillouin. Nous aurions alors

$$
K_{\left(v_{2}-v_{1}\right)}(T)=A\left(\frac{1}{\mathrm{e}^{h v_{1} / k T}-1}-\frac{1}{\mathrm{e}^{h v_{2} / k T}-1}\right)
$$

et l'isochromatique ne dépendrait que de trois paramètres : $A, v_{1}$ et $v_{2}$. Dans le cas de $\mathrm{KBr}$, on connaît très bien les phonons caractéristiques du réseau. Nous n'avons donc qu'un paramètre $\mathrm{A}$ à déterminer expéri- 
mentalement. Il suffira d'un point de l'isochromatique pour déterminer toute la courbe dans cette hypothèse. Nous verrons alors si les points correspondant aux autres températures d'expérimentation se placent bien sur la courbe théorique. L'expérience est intéressante pour résoudre plusieurs problèmes. Les processus de différence sont-ils à deux ou trois phonons ? Quels vecteurs d'onde entrent en jeu ? Y a-t-il des règles de sélection ? Par exemple les transitions $\mathrm{L} \rightarrow \mathrm{L}$ et $\mathrm{T} \rightarrow \mathrm{T}$ sont-elles seules permises comme il est assez probable ?

Nous n'avons eu de résultats assez précis que récemment à cause de la difficulté à mesurer la température d'un échantillon qui n'est pas dans une enceinte isotherme. Nous avons pris, pour $\mathrm{KBr}$, les quatre longueurs d'onde suivantes:

$198 \mu$ correspondant à TA $\rightarrow$ TO $(001)$

$349 \mu$ correspondant à $\mathrm{TA} \rightarrow \mathrm{TO}(111)$

$161 \mu$ correspondant à LA $\rightarrow$ LO $(001)$

$268 \mu$ correspondant à $\mathrm{LA} \rightarrow$ LO (111)

VII. 2 Première véRIFICATION EXPÉRIMENTALE. - La figure 34 donne le spectre de transmission d'un échantillon $e=3 \mathrm{~mm}$, à quatre températures différentes que l'on évalue à $300^{\circ} \mathrm{K}, 100 \mathrm{~K}, 42 \mathrm{~K}$ et $36^{\circ} \mathrm{K}$. On en a tiré (Fig. 33) les quatre isochromes $\lambda=349$ microns ; $\lambda=268$ microns ; $\lambda=198$ microns ; $\lambda=161$ microns donnant $K$ en fonction de $T$ en admettant que $\tau=\tau^{*}(1-R)^{2}$, avec $(1-R)^{2}=70 \%$.

1) $\lambda=349$ microns : $v_{1}=2,20 \times 10^{12} \mathrm{cps}$; $v_{2}=3,06 \times 10^{12} \mathrm{cps} ;$ d'où $n_{1}-n_{2}=0,79$ à $300 \mathrm{oK}$; 0,23 à $100 \circ \mathrm{K} ; 0,056$ à $42{ }^{\circ} \mathrm{K}$ et 0,038 à $36^{\circ} \mathrm{K}$. L'expérience montre qu'à $300{ }^{\circ} \mathrm{K} \tau \simeq 3 \%$, soit $\tau^{*} \simeq 2,7 \%$, d'où $K_{300^{\circ} \mathrm{K}} \simeq 12 \mathrm{~cm}^{-1}$ et $A=17 \mathrm{~cm}^{-1}$.

Par suite :

a) pour $T=100 \circ \mathrm{K}: K=3,9 \mathrm{~cm}^{-1}$;

$$
\tau^{*}=0,31 \text {, or } \tau_{\exp }^{*}=0,49
$$

b) pour $T=42{ }^{\circ} \mathrm{K}: K=0,95 \mathrm{~cm}^{-1}$;

$$
\tau^{*}=0,75 \text {, or } \tau_{\exp }^{*}=0,96
$$

c) pour $T=36^{\circ} \mathrm{K}: K=0,65 \mathrm{~cm}^{-1}$;

$$
\tau^{*}=0,82 \text { or } \tau_{\exp }^{*}=0,99 .
$$

La théorie est bien vérifiée dans la limite des erreurs expérimentales qui sont grandes. L'augmentation de la transparence lorsqu'on refroidit semble toutefois un peu plus grande que prévue par des processus à deux phonons, peut-être que des processus à trois phonons interviennent déjà.
2) $\lambda=268$ microns : $v_{1}=2,68 \times 10^{12} \mathrm{cps}$; $v_{2}=3,80 \times 10^{12} \mathrm{cps} ;$ d'où $n_{1}-n_{2}=0,70$ à $3000^{\circ} \mathrm{K}$; 0,19 à $100 \circ \mathrm{K} ; 0,037$ à $42^{\circ} \mathrm{K} ; 0,023$ à $36^{\circ} \mathrm{K}$. L'expérience donne $\tau_{100^{\circ} \mathrm{K}}=17 \%$, soit $\tau^{*}=19 \%$ et $K=5,5 \mathrm{~cm}^{-1}$. D'où $A=29 \mathrm{~cm}^{-1}$.

Par suite :

a) pour $T=300^{\circ} \mathrm{K}: K=20 \mathrm{~cm}^{-1}$,

$$
\tau^{*}=0,2 \% \text { or } \tau_{\exp }^{*}<0,5 \%
$$

b) pour $T=42{ }^{\circ} \mathrm{K}: K=1,07 \mathrm{~cm}^{-1}$,

$$
\tau^{*}=73 \% \text {, or } \tau_{\exp }^{*} \simeq 91 \%
$$

c) pour $T=36 \circ \mathrm{K}: K=0,66 \mathrm{~cm}^{-1}$,

$$
\tau^{*}=82 \% \text {, or } \tau_{\exp }^{*} \simeq 100 \% .
$$

L'accord avec l'expérience est bon, peut-être y a-t-il une erreur systématique qui augmente la transmission à basse température.

3) $\lambda=198$ microns : $v_{1}=2,82 \times 10^{12} \mathrm{cps}$; $v_{2}=4,34 \times 10^{12} \mathrm{cps} ;$ d'où $n_{1}-n_{2}=0,65$ à $300{ }^{\circ} \mathrm{K}$; 0,21 à $100^{\circ} \mathrm{K} ; 0,035$ à $42^{\circ} \mathrm{K}$ et 0,02 à $36^{\circ} \mathrm{K}$. L'expérience montre qu'à $42{ }^{\circ} \mathrm{K}, \tau=56 \%$ soit $\tau^{*} \simeq 62 \%$, d'où $K=1,6 \mathrm{~cm}^{-1}$ et $A=46 \mathrm{~cm}^{-1}$.

Par suite :

a) pour $T=100{ }^{\circ} \mathrm{K}: K=9,6 \mathrm{~cm}^{-1}$;

$$
\tau^{*}=5,6 \% \text {, or } \tau_{\exp }^{*}=2,5 \%
$$

b) pour $T=36^{\circ} \mathrm{K}: K=0,9 \mathrm{~cm}^{-1}$;

$$
\tau^{*}=76 \%, \tau=68 \% \text {, or } \tau_{\exp }^{*}=75 \% \pm 7 \% \text {. }
$$

Là encore la théorie est bien vérifiée. Peut-être les transmissions mesurées à basse température sont-elles un peu trop grandes du fait d'une erreur systématique.

4) $\lambda=161$ microns : $v_{1}=2,5 \times 10^{12} \mathrm{cps}$, $v_{2}=4,02 \times 10^{12} \mathrm{cps} ; n_{1}-n_{2}=1,38$ à $300 \mathrm{oK}$, 0,39 à $100{ }^{\circ} \mathrm{K} ; 0,083$ à $42^{\circ} \mathrm{K}$ et $0,056^{\circ}$ à $36^{\circ} \mathrm{K}$. L'expérience donne $\tau_{422^{\circ} \mathrm{K}}=29 \%$, d'où $K=3 \mathrm{~cm}^{-1}$ et $A=36 \mathrm{~cm}^{-1}$.

Par suite :

a) Pour $T=100 \mathrm{~K}: K \simeq 14 \mathrm{~cm}^{-1}, \tau=1,3 \%$, or $\tau_{\text {mesuré }} \simeq 0,5 \%$,

b) pour $T=36{ }^{\circ} \mathrm{K}: K \simeq 9 \mathrm{~cm}^{-1}, \quad \tau=39 \%$, or $\tau_{\text {mesuré }}=45 \%$.

L'accord avec l'expérience est bon.

VII. 3 DeUXIÈME VÉRIFICATION EXPÉRIMENTALE. Nous avons essayé d'augmenter la précision en fixant la position du réseau sur une longueur d'onde fixe. 
On élimine les erreurs provenant de la constante de temps souvent trop longue par rapport à la vitesse de rotation du réseau. Toutefois on n'élimine pas les erreurs provenant de la dérive de la source et de l'électronique, puisque la transmission de la lame doit être comparée à celle de l'appareil, sans échantillon sur le trajet optique. Nous ne disposions malheureusement plus du premier échantillon. Le nouveau cristal s'est montré plus opaque, particulièrement à basse température où la transmission n'atteint pas $55 \%$ à 349 microns.

\section{TABLEAU II}

$$
\lambda=349 \text { microns (TA } \rightarrow \text { TO) } ; A=21 \mathrm{~cm}^{-1} \text {. }
$$

\begin{tabular}{|c|c|c|c|c|c|c|}
\hline$T \mathrm{oK}$ & $25^{\circ}$ & $32^{\circ}$ & $40^{\circ}$ & $90^{\circ}$ & $100^{\circ}$ & $300^{\circ}$ \\
\hline$\tau_{\text {exp }}$ & 0,57 & 0,57 & 0,535 & 0,30 & $\mathrm{C}, 255$ & 0,035 \\
\hline$\tau_{\exp }^{*}$ & 0,81 & 0,81 & 0,76 & 0,43 & 0,36 & 0,05 \\
\hline$K_{\text {exp }}$ & 1 & 1 & 1,35 & 4,2 & 5,05 & 14 \\
\hline$K_{\text {calc }}$ & 0,25 & 0,59 & 1,1 & 4,2 & 4,8 & 16,5 \\
\hline$\tau_{\text {calc. }}^{*}$ & 0,95 & 0,89 & 0,80 & 0,43 & 0,38 & 0,03 \\
\hline
\end{tabular}

TABleaU III

$\lambda=268$ microns $(\mathrm{LA} \rightarrow \mathrm{LO}): A=32 \mathrm{~cm}^{-1}$.

\begin{tabular}{|c|c|c|c|c|c|c|c|}
\hline$T \circ \mathrm{K}$ & $25^{\circ}$ & $32^{\circ}$ & $45^{\circ}$ & $90^{\circ}$ & $103^{\circ}$ & $200^{\circ}$ & $300^{\circ}$ \\
\hline$\tau_{\exp }$ & 0,55 & 0,53 & 0,40 & 0,22 & 0,20 & 0,05 & 0,01 \\
$\tau_{\text {exp }}^{*}$ & 0,79 & 0,76 & 0,57 & 0,31 & 0,28 & 0,08 & 0,01 \\
$K_{\text {exp }}$ & 1,15 & 1,35 & 2,8 & 5,8 & 6,25 & 12,8 & 21,3 \\
$K_{\text {calc. }}$ & 0,16 & 0,51 & 1,36 & 5,05 & 6,3 & 13,7 & 21,4 \\
$\tau_{\text {calc. }}^{*}$ & 0,97 & 0,90 & 0,76 & 0,35 & 0,28 & 0,06 & 0,01 \\
\hline
\end{tabular}

TABLEAU IV

$\lambda=161$ microns $(\mathrm{LA} \rightarrow \mathrm{LO}): A=37 \mathrm{~cm}^{-1}$.

\begin{tabular}{|c|l|l|l|c|c|}
\hline$T$ ०K & $25^{\circ}$ & $31^{\circ}$ & 370 & $90^{\circ}$ & $100^{\circ}$ \\
\hline$\tau_{\exp }$ & 0,41 & 0,3 & 0,21 & 0,07 & 0,05 \\
$\tau_{\text {exp }}^{*}$ & 0,58 & 0,43 & 0,30 & 0,10 & 0,07 \\
$K_{\text {exp }}$ & 2,7 & 4,2 & 6 & 11,5 & 12,9 \\
$K_{\text {calc. }}$ & 0,63 & 1,3 & 2,25 & 12,3 & 14,3 \\
$\tau_{\text {calc. }}^{*}$ & 0,88 & 0,77 & 0,64 & 0,08 & 0,06 \\
\hline
\end{tabular}

VIII. Processus de différence à trois phonons. Il est évident que si les transitions $\mathrm{T} \rightarrow \mathrm{L}$ sont interdites, les processus à deux phonons ne peuvent jouer au-dessus d'une certaine longueur d'onde $\lambda_{\mathrm{c}}$. Stolen a montré qu'au-delà, l'absorption varie comme $T^{2}$ et correspond à des processus de différence à trois phonons.

Au-dessous de $\lambda_{\mathrm{c}}$, les processus à trois phonons peuvent jouer un certain rôle et expliquer que les variations observées de $K$ avec $T$ soient très légèrement plus grandes que prévu, dans la première série de mesures.

IX. Absorption induite par les impuretés. - Lorsque, par refroidissement, l'absorption générale des halogénures alcalins dans l'infrarouge lointain disparaît, nous avons observé un grand nombre de raies d'absorption, d'autant plus intéressantes que l'infrarouge lointain a longtemps eu la mauvaise réputation d'être pauvre en transitions, si ce n'est entre les différents états rotationnels d'un gaz polaire. Nous avons trouvé ces bandes dans la plupart des cristaux du commerce [3], complètement transparents par ailleurs dans l'infrarouge moyen où ils sont utilisés comme prismes ou fenêtres. Par contre, les raies à 99,120 et $140 \mu$ n'apparaissent pas dans un cristal réalisé par fusion d'un produit Merck ultra pur pour analyses [3]. Seule, la raie à $107 \mu$ est toujours présente. Dans plusieurs cas $(\mathrm{KBr}, \mathrm{NaI})$, ces raies varient d'intensité d'un échantillon à l'autre, mais restent fixes en position, et correspondent très sensiblement à des fréquences où les courbes de densité de phonons $\mathrm{d} N / \mathrm{d} \omega$ présentent un maximum [3]. Nous nous sommes proposés de voir quels défauts, ou quelles limpuretés permettaient cette activité. Les centres $F$ produits par irradiation de $\mathrm{KBr}$ aux rayons $\beta$ (environ $2 \times 10^{16}$ électrons dont les courbes énergétiques présentent des pics à 0,58 et 2,2 MeV), par la valeur de potassium, ou l'irradiation aux neutrons $\left(1,9 \times 10^{18} \mathrm{~cm}^{-2}\right.$ neutrons thermiques; $6,87 \times 10^{17} \pm 5 \%$ neutrons rapides $\left.[1 \mathrm{MeV}]\right)$ colorent en bleu le cristal, conduisent à une absorption générale dans l'infrarouge lointain, mais sont inefficaces pour faire apparaître les bandes d'impuretés à $99 ; 120$ et 140 microns.

Tous les ions que nous avons essayé d'incorporer dans le réseau conduisent à une absorption générale sans structure nette, à l'exception de l'ion sodium, qui se montre seul efficace pour faire apparaître les pics à $99 ; 120$ et $140 \mu$, qui correspondent très sensiblement aux fréquences des phonons TO, LA et TA à l'extrémité de la zone de Brillouin [10]. 


\section{Bibliographie}

[1] Hadni (A.), Claudel (J.), Gerbaux (X.), Morlot (G.) et MuniER (J. M.), Sur le comportement différent des cristaux et des verres dans l'absorption de l'infrarouge lointain $(40-1500 \mu)$ à la température de l'hélium liquide. Appl. Opt., 1965, 4, 487.

[2] Buz (H.), GeNzel (L.) und HAPP (H.), Zur Ultrarotdispersion der Alkali-Halogenide I. die Deutung der Spektren nach der Theorie von Born und Huang. Z. Physik, 1960, 160, 535.

[3] Hadni (A.), Morlot (G.), Brehat (F.) et Strimer (P.), Sur le rôle des impuretés pour activer optiquement dans l'infrarouge lointain les ondes élastiques des cristaux ioniques, C. R. Acad. Sc., $1965,261,2605$.

Hadni (A.), Morlot (G.), Gerbaux (X.), Chanal (D.), Brehat (F.) et Strimer (P.), Absorption induite dans l'infrarouge lointain par les impuretés et les défauts d'un solide, C. R. Acad. Sc., 1965, 260, 4973.

[4] Jones (G. O.), Martin (D. H.) Mawer (P. A.) and
PERRY (C. H.), Spectroscopy at extreme infra-red wevelengths. II. the lattice resonances of ionic crystals, Proc. Roy. Soc., 1961, A 261, 10.

[5] SToLen (R.), Far infrared absorption in alkali halide crystals. These (Berkeley 1964).

[6] Sievers (A. J.), Far infrared resonance states in silveractivated potassium halide crystals, Phys. Rev. Letters, 1964, 13, 310.

[7] Dryden (J. S.) and Meakins (R. J.), Dielectric relaxation processes in lithium, sodium and potassium halides, Communication personnelle, 1957.

[8] Mrrsurshr (A.), The optical properties of cadmium telluride in the far infrared region, J. Phys. Soc. Japan, 1961, 16, 533.

[9] Mrtsuishi (A.), Far infrared measurements on lattice vibration spectra of several diatomic crystals, communication personnelle (séminaire sur la spectroscopie dans l'infrarouge lointain, Colombus, Ohio, septembre 1965).

[10] Hadni (A.), Morlot (G.), Bréhat (F.), C. R. Acad. Sc., janvier 1967.

[11] Lowndes (R. P.), Physics Letters, 1966, 21, 26. 